\title{
XXIII. Note on the measure of the intensity of sound
}

\section{R.H.M. Bosanquet}

To cite this article: R.H.M. Bosanquet (1880) XXIII. Note on the measure of the intensity of sound, Philosophical Magazine Series 5, 9:55, 174-177, DOI: 10.1080/14786448008626822

To link to this article: http://dx.doi.org/10.1080/14786448008626822

曲 Published online: 08 Jun 2010.

Submit your article to this journal ๘

Џll Article views: 2

Q View related articles $\sqsubset$ 
(3) Directly as the mutual attraction of the two conductors that limit the field, or

(4) Directly as the electric tension of the dielectric, a quantity that was conceived long ago very clearly by Faraday, and introduced afterwards definitely into the Mathematical Theory of Electricity by Professor Clerk Maxwell.

Faraday's and Clerk Maxwell's views as to the action of the dielectric in the transmission of electrostatic force, and as to the state of molecular constraint that is associated with and essential to that action, are very strongly confirmed by the new facts of electro-optics. The dioptric action of an electricallycharged medium is closely related to the electric stress of the medium, the axis of double refraction coinciding in every case with the line of electric tension, and the intensity of double refraction varying, certainly in $\mathrm{CS}_{2}$ and probably in all other dielectrics, directly and simply as the intensity of the tension.

Glasgow, January 22, 1880.

XXIII. Note on the Measure of the Intensity of Sound. By R. H. M. Bosanquet, Fellow of St. John's College, Oxford. To the Editor's of the Philosophical Magazine and Journal. GenTlemen,

T AM happy to say that my proposal for the establishment 1 of an acoustic laboratory* is in a fair way to be realized, and I hope shortly to get to work. But I reserve all account of this for the present.

The object of this note is to present certain considerations as to the expression of the measure of sound-intensities, with a deduction of Fechner's law in a manner which I do not remember to have seen before. The point was suggested to me by a criticism, in which it appeared to be supposed that the measurement of differences of sensation arising from differences of intensity by the ratio of the mechanical intensities was inconsistent with Fechner's law.

The first and simplest hypothesis concerning the difference of the sensations due to two mechanical intensities is, that the difference of the numbers which express the measure of the sensation is proportional to the difference of the mechanical intensities. (The variations due to difference of pitch are throughout disregarded, as that question forms a distinct

* Phil. Mrg. Oct. 1879. 
branch of the subject.) It is easy to prove, from facts of ordinary knowledge, that this hypothesis is impossible. For instance, a single diapason stop of soft quality, in the organ, may be taken to represent a definite mechanical intensity. If this be added to a mechanical intensity of less, or not very much greater amount, the addition produces a finite difference of sensation. If, however, the full organ is being used, so that the existing mechanical intensity is very much greater than that added by the single soft stop, the addition of the latter does not affect the sensation at all. The first hypothesis therefore falls to the ground.

Similar conditions may be applied to any definite loud and soft sounds; but the above is sufficient to establish the point.

The second hypothesis in point of simplicity would appear to be, that the difference of the numbers which express the measure of the sensation is proportional to the ratio of the mechanical intensities. This is an hypothesis often practically employed in a vague sort of way. I shall endeavour to trace it to its consequences, and show that it is necessary that the measure of sensation thus defined should be connected with another measure of sensation. The second measure introduced is that in which small differences of mechanical intensity correspond to small differences of sensation; and this latter measure is related to the mechanical intensity, according to Fechner's law.

Let $\lambda$ be the measure of sensation, differences of which are proportional to the ratio of the mechanical intensities, so that

Let

$$
\lambda_{1}-\lambda_{0}=k \frac{\mathrm{I}_{1}}{\mathrm{I}_{0}} \cdot \text {. . . . . }
$$

$$
\begin{array}{ll}
\lambda_{1}=\lambda+\Delta \lambda, & \mathrm{I}_{1}=\mathrm{I}+\Delta \mathrm{I}, \\
\lambda_{0}=\lambda, & \mathrm{I}_{0}=\mathrm{I} .
\end{array}
$$

Then (i) becomes

$$
\begin{aligned}
\Delta \lambda & =k \frac{\mathrm{I}+\Delta \mathrm{I}}{\mathrm{I}} \\
& =k\left(1+\frac{\Delta \mathrm{I}}{\mathrm{I}}\right) . \quad \cdot . . . \cdot
\end{aligned}
$$

If we diminish $\Delta \mathrm{I}$ indefinitely, $\Delta \lambda$ tends to the value $k$, which cannot vanish consistently with the fundamental assumption. $\lambda$ cannot, therefore, be such a measure of sensation as we ordinarily recognize, since differences of sensation must ultimately diminish indefinitely with differences of the mechanical intensity. 
Taking logarithms in (ii),

$$
\begin{aligned}
\log _{e} \Delta \lambda & =\log _{e} k+\log _{e}\left(1+\frac{\Delta \mathrm{I}}{\mathrm{I}}\right) \\
& =\log _{e} k+\frac{\Delta \mathrm{I}}{\mathrm{I}}
\end{aligned}
$$

ultimately, when $\frac{\Delta I}{I}$ is small. If then we put

$$
\begin{aligned}
& \Delta \sigma=\log _{e} \Delta \lambda \text { and } k=1, \\
& \qquad \Delta \sigma=\frac{\Delta \mathrm{I}}{\mathrm{I}}, . . . . . .
\end{aligned}
$$

and the differences of the new measure of sensation $(\sigma)$ and the mechanical intensity vanish together. This equation (iii) is the basis of Fechner's law.

Resuming the argument. The hypothesis that differences of a measure of sensation are proportional to the ratios of mechanical intensity is impossible, totidem verbis ; but the differences of the measure in question are the logarithms of the differences of another measure, which satisfies the conditions of observation, and leads to Fechner's law.

A third assumption, which may perhaps be stated separately, is that the ratio of the sensations is proportional to the ratio of the intensities-that is,

$$
\frac{\lambda_{1}}{\bar{\lambda}_{0}^{-}}=k \frac{\mathrm{I}_{1}}{\mathrm{I}_{0}^{-}}
$$

and, with the same notation as before,

$$
1+\frac{\Delta \lambda}{\lambda}=k\left(1+\frac{\Delta \mathrm{I}}{\mathrm{I}}\right)
$$

If the differences are to vanish simultaneously,

and

$$
k=1 \text {, }
$$

where

$$
\lambda=k^{\prime} \mathrm{I}
$$

$$
k^{\prime}=\frac{\lambda_{0}}{\bar{I}_{0}}
$$

Whence it follows that the differences of sensation $(\Delta \lambda)$ are proportional to the differences of mechanical intensity $(\Delta \mathrm{I})$; but this was shown to be untrue in connexion with the first hypothesis. This hypothesis is therefore really identical with that first made, and falls with it.

Of these, the simplest hypotheses that can be made on this subject, the only one which satisfies the elementary conditions, 
is that which leads to Fechner's law. It is possible that this law may not be accurately and rigorously true; that would only be to say that our fundamental hypotheses err in the same manner, which is not at all improbable. But until experiments of a far more careful and extensive character than any yet made come to decide the question, we may take Fechner's law to be highly probable.

This law will always probably be best led up to by the physiological reasoning commonly employed. But I have thought it not useless to examine the consequences of the various elementary assumptions as to the measure of the sensation of sound, from a different point of view.

P.S.-Since the above was written, my attention has been drawn to an experimental verification of Fechner's law, for the sounds produced by falling woights (Carl Nörr, Zeitschrift für Biologie, 1879, p. 297).

XXIV. On the Mean free Path of the Molecules. $B y$ N. D. C. HodGes*.

THE free path of a molecule is dependent on the amount 1 of obstruction it meets with, or the density of the medium. O. E. Meyer gives for the mean free path (on page 308 of his Kinetische Theorie der Gase), $\mathrm{L}=\frac{1}{\pi \sqrt{2} \mathrm{~N} \zeta^{2}}$. Here $\mathrm{N}$ is the number of molecules in the unit volume.

I consider the length of path in a medium of variable density. At the surface of a liquid, if there is no sharp transition from the liquid to the gaseous state, we shall have a succession of layers of less and less dense vapours, from where there is liquid to the surrounding atmosphere. The layers $\mathrm{V}$ are what I refer to. The depth of these vapours is, of course, much magnified.

I propose to find the pressure upon the particle $p$, when the surface of the liquid is plane and when it is spherical. Taking the molecules moving with any definite velocity, they will reach $p$ and give it an impulse when they are at a distance from $p$ less than their mean free path. Now the molecules from below come from denser layers than those from above. A greater number will come from below than from above; there will be a tendency to drive $p$ upward. To find this tendency, we must find how much denser the lower layers, from

* Communicated by the Author. 